\title{
Localization transition of stiff directed lines in random media
}

\author{
Horst-Holger Boltt $2^{*}$ and Jan Kierfeld \\ Physics Department, TU Dortmund University, 44221 Dortmund, Germany
}

(Dated: June 20, 2021)

\begin{abstract}
We investigate the localization of stiff directed lines with bending energy by a short-range random potential. Using perturbative arguments, Flory arguments, and a replica calculation, we show that a stiff directed line in $1+\mathrm{d}$ dimensions undergoes a localization transition with increasing disorder for $d>2 / 3$. We demonstrate that this transition is accessible by numerical transfer matrix calculations in $1+1$ dimensions and analyze the properties of the disorder-dominated phase. On the basis of the two-replica problem, we propose a relation between the localization of stiff directed lines in $1+d$ dimensions and of directed lines under tension in $1+3 d$ dimensions, which is strongly supported by identical free energy distributions. This shows that pair interactions in the replicated Hamiltonian determine the nature of directed line localization transitions with consequences for the critical behavior of the Kardar-Parisi-Zhang (KPZ) equation. Furthermore, we quantify how the persistence length of the stiff directed line is reduced by disorder.
\end{abstract}

PACS numbers: 05.40.-a,64.70.-p,64.60.Ht,61.41.+e

Directed lines (DLs) in random media or the more general problem of random elastic manifolds are one of the most important model systems in the statistical physics of disordered systems [1]. DLs in random media are related to important non-equilibrium statistical physics problems such as stochastic growth, in particular the KPZ equation 2, Burgers turbulence, or the asymmetric simple exclusion model (ASEP) [3]. Furthermore, there are many applications such as kinetic roughening [3, pinning of flux lines 4, 5], random magnets, or wetting fronts [1.

DLs in $D=1+d$-dimensional random media exhibit a disorder-driven localization transition for dimensions $d>2$, which has been studied numerically for dimensions up to $d=3[6-9$. In the context of the KPZ equation, it is a long-standing open question whether there exists an upper critical dimension, where the critical behavior at the localization transition is modified (for a recent discussion, see, e.g., [10]). We will address this question from a new perspective by revealing a relation between DLs in $1+3 d$ dimensions and stiff directed lines (SDLs) in $1+d$ dimensions in a random medium, which we validate for $d=1$ by numerical simulations. We define SDLs as lines with preferred orientation and no overhangs with respect to this direction, but a different elastic energy penalizing curvature instead of stretching, cf. Fig. 1. We investigate their disorder-induced localization transition and the scaling properties of conformations in the disordered phase. Because the proposed relation between DLs and SDLs is based on return probabilities of pairs of replicas our results suggest that the critical properties of DLs in a random potential and, thus, of the KPZ equation are determined by the corresponding two replica problem.

Model of the stiff directed line. The configuration of a directed line, i.e. one without overhangs or loops and

*horst-holger.boltz@udo.edu

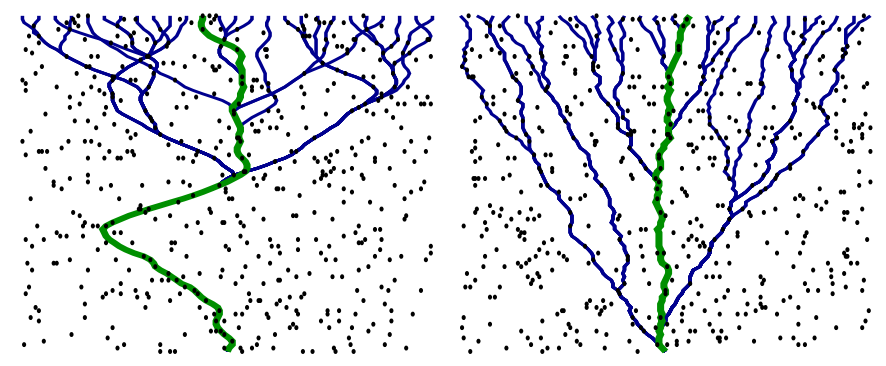

FIG. 1: (color online) Paths with lowest energy for given ending states (top, globally optimal path thicker) for (a) the stiff and (b) the tense directed line (right). The dots mark favorable regions of the Gaussian random potential $V$ (realizations of the quenched disorder are not identical in a and b).

without inextensibility constraint, can be parametrized by $(x, \mathbf{z}(x))(0 \leq x \leq L)$ with a $d$-dimensional displacement $\mathbf{z}(x)$ normal to its preferred orientation. Each configuration of a SDL is associated with an energy

$$
\mathcal{H}=\int_{0}^{L} \mathrm{~d} x\left[\frac{\kappa}{2}\left(\partial_{x}^{2} \mathbf{z}(x)\right)^{2}+V(x, \mathbf{z}(x))\right],
$$

where the first term is the bending energy (to leading order in $\mathbf{z}$ ). The second term is the disorder energy with a Gaussian distributed quenched random potential $V(x, \mathbf{z}(x))$ with zero mean $\bar{V}=0$ and short-range correlations $\overline{V(x, \mathbf{z}) V\left(x^{\prime}, \mathbf{z}^{\prime}\right)}=g^{2} \delta^{d}\left(\mathbf{z}-\mathbf{z}^{\prime}\right) \delta\left(x-x^{\prime}\right)$ [35.

The SDL model (1) is an approximation to the socalled worm-like chain or Kratky-Porod model [11, 12] $\mathcal{H}_{\mathrm{WLC}}=\int_{0}^{L} \mathrm{~d} s\left[\frac{\kappa}{2}\left(\partial_{s}^{2} \mathbf{r}(s)\right)^{2}+V(\mathbf{r}(s))\right]$ which is a common model for semiflexible polymers, such as DNA or cytoskeletal filaments like F-actin. The chain is parametrized in arc length, leading to a local inextensibility constraint $\left|\partial_{s} \mathbf{r}(s)\right|=1$, which is hard to account for, both numerically and analytically [13, 14. The approximation (11) only applies to a weakly bent semiflexible polymer on length scales below the so-called persistence 
length, which is [36] $L_{p}=(D-1) \kappa / 2 T$ for thermally fluctuating semiflexible polymers [14]. Above the persistence length, a semiflexible polymer loses orientation correlations and starts to develop overhangs. Also in a quenched random potential the SDL model describes semiflexible polymers in heterogeneous media, only as long as tangent fluctuations are small such that overhangs can be neglected, which is the case below a disorder-induced persistence length, which we will derive below.

We consider the SDL model also in the thermodynamic limit beyond this persistence length, because we find evidence for a relation to the important problem of DLs in a random medium in lower dimensions. This relation is based on replica pair interactions and shows that pair interactions also determine the nature of the DL localization transition. Moreover, this relation can make the DL transition in high dimensions computationally accessible. We will now outline the idea behind this relation.

Relation to directed lines. The difference between SDLs and DLs [1] is the second derivative in the first bending energy term in eq. (1) for SDLs, which differs from the tension or stretching energy $\sim \int d x\left(\partial_{x} \mathbf{z}(x)\right)^{2}$ of DLs. This results in different types of energetically favorable configurations (see Fig. 11): large perpendicular displacements $\mathbf{z}$ are not unfavorable as long as their "direction" does not change. Displacements $\mathbf{z}$ are characterized by the roughness exponent $\zeta$, which is defined by $\overline{\left\langle z^{2}(L)\right\rangle} \sim L^{2 \zeta}$. The thermal roughness is $\zeta_{t h, \tau}=1 / 2$ for DLs and $\zeta_{t h, \kappa}=3 / 2$ for SDLs. Here and in the following we use subscripts $\tau$ (tension) and $\kappa$ (bending stiffness) to distinguish between the two systems.

Although typical configurations are quite different, a SDL subject to a short-ranged (around $z=0$ ) attractive potential $V(\mathbf{z})$ can be mapped onto a DL in high dimensions $d^{\prime}=3 d[15,16]$. This equivalence is based on the probability that a free line of length $L$ starting at $(\mathbf{z}(0)=0)$ "returns" to the origin, i.e., ends at $\mathbf{z}(L)=0$. This return probability is characterized by a return exponent $\chi: \operatorname{Prob}(\mathbf{z}(L)=0) \sim L^{-\chi}$. For DLs, which are essentially random walks in $d$ transverse dimensions, the return exponent is $\chi_{\tau}=d / 2$ [17, whereas it is $\chi_{\kappa}=3 d / 2$ for a SDL (after integrating over all orientations of the end) [18; they are related to the roughness exponents by $\chi=\zeta d[16]$. The return exponent governs the binding to a short-range attractive potential or, equivalently, the binding of two lines interacting by such a potential [1517. The relation $\chi_{\tau}(3 d)=\chi_{\kappa}(d)$ implies that the binding transition of two DLs in $3 d$ dimensions maps onto to the binding transition of two SDLs in $d$ dimensions.

In the replica formulation of line localization problems such as (1), the random potential gives rise to a shortrange attractive pair interaction (see below). Furthermore, the critical temperature $T_{c, \tau}$ for a DL in a random potential is believed to be identical to the critical temperature $T_{2, \tau}$ for a system with two replicas [9, 19]. One aim of this work is to generalize this conjecture by demonstrating that the $d \rightarrow 3 d$ analogy between DLs and SDLs in a random potential holds for the entire free energy dis-

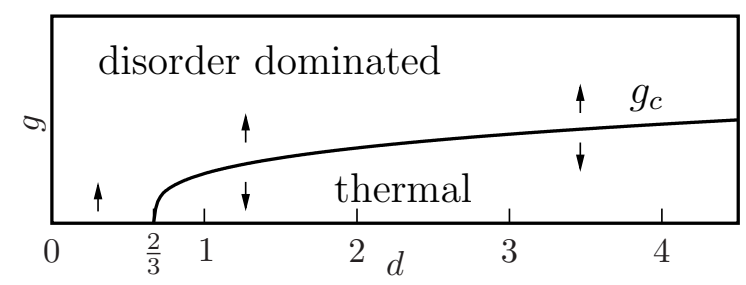

FIG. 2: Sketch of the phase diagram as implied by Florytype arguments. The arrows indicate the flow of the disorder "strength" $g$ under renormalization.

tribution. Because this analogy is rooted in the binding transition of replica pairs, we can conclude that critical properties of the localization transition are determined by pair interactions in the replicated Hamiltonian, which has been previously suggested in Refs. [20, 21]. Moreover, it has been proposed that pair interactions can be used to formulate an order parameter of the disorder-driven localization transition of DLs in terms of the overlap $q \equiv \lim _{L \rightarrow \infty} \overline{\frac{1}{L} \int_{0}^{L} \mathrm{~d} x \delta\left(z_{1}(x)-z_{2}(x)\right)}[22-24$, i.e., the average number of sites per length, that two lines in the same realization of the disorder have in common. Localization by disorder gives rise to a finite value of the pair overlap $q$.

Scaling analysis. We start with a scaling analysis of SDL behavior in disorder by a Flory-type argument. For displacements $\sim z$ the bending energy in eq. (1) scales as $E_{b} \sim \kappa z^{2} L^{-3}$, which also leads to $\left\langle z^{2}\right\rangle \sim L^{3} / L_{p}$ and the thermal roughness exponent $\zeta_{t h, \kappa}=3 / 2$. The disorder energy in eq. 11 scales as $E_{d} \sim g \sqrt{L z^{-d}}$. Using the unperturbed thermal roughness in the disorder energy we get $E_{d} \sim L^{(2-3 d) / 4}$, from which we conclude that the disorder is relevant below a critical dimension $d<d_{c, \kappa}=2 / 3$. For $d>d_{c, \kappa}=2 / 3$ and, thus, in all physically accessible integer dimensions, the SDL should exhibit a transition from a thermal phase for low $g$ to a disorder dominated phase above a critical value $g_{c}$ of the disorder (see Fig. 2). In the disorder dominated phase, the SDL becomes localized and assumes a roughened configuration, see Fig. 1 .

Balancing the Flory estimates, $E_{b} \sim E_{d}$, gives the roughness $z \sim L^{\zeta_{F l}}$, if disorder is relevant. This leads to $\zeta_{F l, \kappa}=7 /(4+d)$, which is applicable below the critical dimension $d<d_{c, \kappa}$, where $\zeta_{F l, \kappa}>\zeta_{t h, \kappa}$. Above the critical dimension, the Flory result would give $\zeta_{F l, \kappa}<\zeta_{t h, \kappa}$, which contradicts a roughening of the SDL as it adjusts to the random potential. Furthermore, the exponent $\omega$ related to the energy fluctuations via $\Delta F \sim L^{\omega}$ would be negative, since the scaling of the energy implies a general scaling relation $\omega=2 \zeta_{\kappa}-3$ (note that we do not subscript $\omega$ as we believe $\omega_{\tau}=\omega_{\kappa}$, see below). An exponent $\omega<0$ contradicts the existence of large disorder-induced free energy fluctuations in the low-temperature phase [25, 26], for which there is also strong numerical evidence 7.9$]$. We conclude that this kind of argument is not applicable 
above the critical dimension. The same problems occur in Flory arguments for DLs for $d>d_{c, \tau}=2$ as well as in, for example, functional renormalization group analysis 27] for $d \gtrsim 2.5$. We conclude that, in contrast to DLs, it is sufficient to study $1+1$ dimensional SDLs in numerical transfer matrix calculations in order to explore the properties of their localization transition such that we focus on this case in the following.

Variation in replica space. To go beyond scaling arguments we use the replica technique 28 following the treatment of directed manifolds [29, the results of which we summarize briefly in the following. We write the averaged and replicated partition function as $\overline{Z^{n}}=\prod_{\alpha}\left(\int \mathcal{D} z_{\alpha}\right) \exp \left(-\beta \mathcal{H}_{\text {rep }}\right)$ with the replica Hamiltonian in Fourier space $\mathcal{H}_{\text {rep }}=$ $\frac{1}{2 L} \sum_{\alpha=1}^{n} \sum_{k}\left(\kappa k^{4}+\mu\right) z_{\alpha}^{2}-\frac{\beta g^{2}}{2} \sum_{\alpha, \beta=1}^{n} \int_{0}^{L} \mathrm{~d} x f_{\lambda}\left(z_{\alpha \beta}^{2}\right) \quad$ where $z_{\alpha \beta} \equiv z_{\alpha}-z_{\beta}$ and with an attractive potential $f_{\lambda}(z)$ of range $\lambda$. As mentioned before, $\mathcal{H}_{\text {rep }}$ is related to a pair binding problem: in the limit $\lambda \approx 0$ the second term becomes $-\frac{\beta g^{2}}{2} \sum_{\alpha, \beta} \int_{0}^{L} \mathrm{~d} x \delta\left(z_{\alpha}-z_{\beta}\right)$.

Using variation in replica space we find one-step replica symmetry breaking for $d>d_{c, \kappa}$ and can show that there is no localized solution unless the potential strength $g$ and correlation length $\lambda$ are above finite values. We interpret this as an indication for the existence of a critical disorder strength or a critical temperature for $d>d_{c, \kappa}$.

Numerical results. More progress is possible by extensive numerical studies using the transfer matrix method [1, 30, both for $T=0$ (see Fig. 1) and for $T>0$. The transfer matrix element for a segment of a SDL with length $\Delta L=1$ starting at $z$ with orientation $\mathrm{d} z / \mathrm{d} x=v$ and ending at $z^{\prime}$ with $v^{\prime}$ follows from a discretization of (1) as $\Delta E=\kappa / 2\left(v-v^{\prime}\right)^{2}+V\left(z^{\prime}\right)$, where we have chosen $z^{\prime}=z+v^{\prime}$ to simplify the transfer matrix. For the sake of simplicity we choose $g=1$ and vary the temperature.

In order to test the relation between DLs and SDLs, we compare free energy fluctuations for DLs in $1+3$ and SDLs in $1+1$ dimensions. Previous direct numerical studies of DLs in $1+3$ dimensions found an exponent $\omega \approx 0.18$ [8, 9] in the low temperature phase, the most precise value from kinetic roughening studies is $\omega \approx 0.186$ [31]. We compare these values with own numerical transfer matrix calculations for SDLs in $1+1$ dimensions. We determine the exponent $\omega$ directly by fitting $\Delta F=\left(\overline{F^{2}}-\bar{F}^{2}\right)^{1 / 2} \propto$ $L^{\omega}$, which gives values $\omega \approx 0.16$ for DLs in $1+3$ dimensions, or by studying the distribution of the free energy shown in Fig. 3a, which is obtained by computing the free energy for every sample and rescaling to zero mean and unit variance, $G_{F}(x)=\operatorname{Prob}((F-\bar{F}) / \Delta F=x)$. The asymptotic behavior of the negative tail of the rescaled free energy distribution for low temperatures, which is of the form $\ln G_{F}(x) \sim-|x|^{\eta}(x<0,|x| \gg 1)$, allows us to determine the energy fluctuation exponent $\omega$ via the Zhang argument [1] giving $\eta=1 /(1-\omega)$. We get $\eta \approx 1.23$ and therefore $\omega \approx 0.19$. These values agree with the literature values for DLs in $1+3$ dimensions.

For direct comparison of the entire free energy distri- butions of a SDL in $1+1$ and a DL in $1+3$ dimensions we simulated both systems and find that the rescaled free energy distributions in the low temperature phases have to be considered identical within numerical accuracy, see Fig. 3a. This strongly supports the relation between DLs in $1+3 d$ dimensions and SDLs in $1+d$ dimensions.

In a third approach, we can calculate $\zeta_{\kappa}$ and $\omega=$ $2 \zeta_{\kappa}-3$ by measuring a "local" version of the roughness exponent 32] $2 \zeta(L)=\log _{5}\left(z^{2}(L) / z^{2}(L / 5)\right)$. The data shown in Fig. 3 3 shows two distinct regimes for high and low temperatures and a significant "dip" around $T=1.4$. For low temperatures, values $2 \zeta_{\kappa} \approx 3.11$ are consistent with $\omega \approx 0.11$. As for DLs [33], it can be argued that $\omega$ should vanish at the transition, resulting in fluctuations of the free energy that scale logarithmically with $L, \Delta F \sim \ln ^{1 / 2} L[9$, resulting in a roughness exponent $\zeta_{\kappa}=3 / 2$. This seems to hold, even though the numerical value for high temperatures is slightly above $\zeta_{\kappa}=3 / 2$, which is strong evidence for a phase transition at $T_{c} \approx 1.4$. We support this by studying the difference of quenched and annealed free energies $\delta F=\bar{F}-\bar{F}_{\text {ann }}$, which should as well scale as $\delta F \sim \ln ^{1 / 2} L$ at the transition. We determined $F_{\text {ann }}$ by simulating a system without disorder and adding the contribution of the annealed potential, $\bar{F}_{\text {ann }}=F_{g=0}-L g^{2} \beta / 2$, see Fig. 3 b.

Finally, we identify an order parameter of the localization transition. For DLs, the disorder-averaged overlap $q=\lim _{L \rightarrow \infty} \overline{\frac{1}{L} \int_{0}^{L} \mathrm{~d} x \delta\left(z_{1}(x)-z_{2}(x)\right)}$ of two replicas has been proposed as order parameter [22, 23. Up to now, it has been numerically impossible to verify this order parameter for DLs in $d>2$ dimensions where a localization transition exists because the relevant $2 d$-dimensional two replica phase space is too large. For SDLs, on the other hand, the transition is numerically accessible already in $1+1$ dimensions and we show that the overlap $q$ is indeed a valid order parameter using an adaptation of the transfer matrix technique from Ref. 23], see Fig. 33. This involves simulating two interacting SDLs, therefore we can only use lengths up to $L=30$ and $10^{3}$ samples. For DLs, it has been found that the overlap at criticality decays as $q \sim L^{\Sigma}$ with $\Sigma=-2 \zeta=-(1+\omega)$ in $d=3$ [22]. This has been extended to finite temperatures yielding $q \sim\left|T-T_{c}\right|^{-\nu \Sigma}$. Indeed, we find a qualitatively similar behavior $q \sim\left|T-T_{c}\right|^{-\beta^{\prime}}$ with an exponent $\beta^{\prime} \approx 1.3-1.4$. Our best estimate for $\Sigma$ is $\Sigma \approx-0.75$. For the correlation length exponent $\nu$ we find values $\nu \approx 2$ compatible with the corresponding problem of DLs 6 , 9, 19]; such that our present results deviate from $\beta^{\prime}=\nu \Sigma$. Because of small simulation lengths $L$ we do not conclude this deviation to be a definite statement against the renormalization group results presented in Ref. 22. Nevertheless, the connection between DLs and SDLs provides the first system to test the proposed order parameter in a localization transition numerically and to determine the otherwise inaccessible exponents $\beta^{\prime}$ or $\Sigma$.

Disorder-induced persistence length. As stated before, the model of a SDL in a random potential de- 

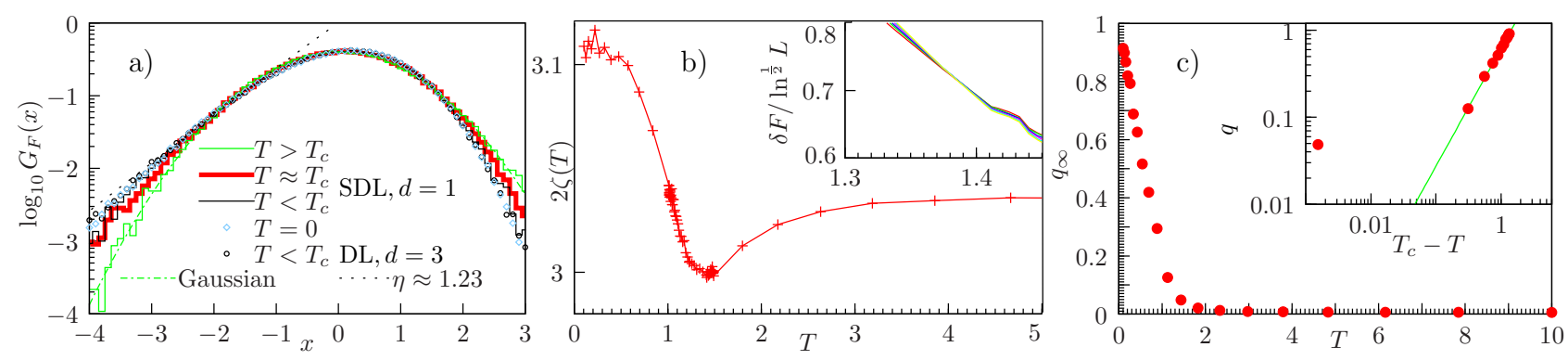

FIG. 3: (color online) a): Rescaled $G_{F}(X)=P((F-\bar{F}) / \Delta F)$ free energy distribution for a stiff directed line (SDL) in $1+1$ dimensions. We show distributions for $T=0$ (light (blue) squares, ground state energy) as well as for three finite temperatures $T<T_{c}$ (black thin solid line), $T \approx T_{c}$ (red, thicker solid line) and $T>T_{c}$ (green, light thin solid line). Results for a directed line (DL) in $1+3$ dimensions are shown (dark circles). b): Local (see text) roughness exponent $2 \zeta_{\kappa}$ as a function of $T$. Deviations from the analytical value $2 \zeta_{\kappa}=3$ at high temperatures indicate numerical problems, nonetheless there is a clear "dip" at $T \approx 1.4$, which we identify as the critical temperature. For low temperatures, we find values consistent with $\omega \approx 0.11$. Inset: Reduced free energy $\delta F=\bar{F}-\bar{F}_{\text {ann }}$ rescaled by $\ln ^{1 / 2} L$ for lengths $L=50,60, \ldots, 100$ as a function of $T$. There is a pseudocrossing around $T \approx 1.38$. c): The overlap order parameter $q$, as a function of $T$. We estimated $q$ from finite lengths using a fit $q_{T}(L)=a(T) / L+q_{\infty}(T)$. Inset: Double-logarithmic plot of the overlap $q$ versus $T_{c}-T$ (with $T_{c}=1.44$ ), the solid line is given by $q \sim\left(T_{c}-T\right)^{-\beta^{\prime}}$ with $\beta^{\prime} \approx-1.36$.

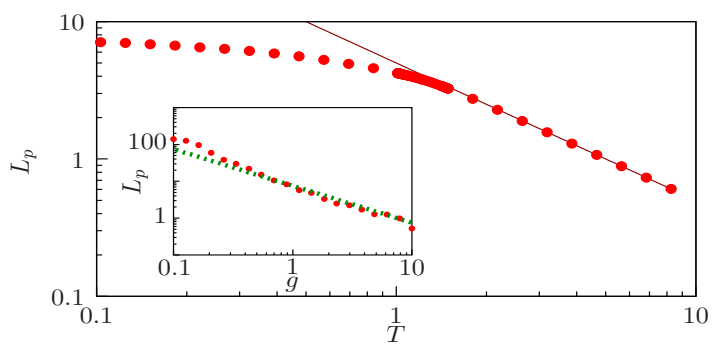

FIG. 4: Disorder-reduced persistence length $L_{p}$ of the SDL for $g=1$. $L_{p}$ matches its thermal value (solid line) for $T>T_{c}$ and is reduced and approximately constant for $T<T_{c}$. Inset: $L_{p}$ at $T=0$ versus the potential strength $g$. The Flory-result $L_{p} \sim g^{-1}$ (dotted line) matches the data.

scribes a worm-like chain in a heterogeneous environment on small length scales $L<L_{p}$ such that overhangs can be neglected, which gives the defining criterion $\overline{\left\langle v^{2}\right\rangle}\left(L=L_{p}\right)=1$ for the persistence length. The line roughens in the low temperature phase, which gives rise to a persistence length decrease as compared to the thermal persistence length $L_{p} \sim \kappa / k_{B} T$. At low temperatures, the Flory-result $z \sim(g / \kappa)^{2 /(4+d)} L^{7 /(4+d)}$ leads to $L_{p} \sim(\kappa / g)^{2 /(3-d)}$. For $d=1$, we determine $L_{p}$ numerically via the above defining criterion, and the data presented in Fig. 4 does indeed show an only weakly temperature-dependent disorder-reduced persistence length for $T<T_{c}$. The $T=0$ results match the Flory-result $L_{p} \sim \kappa / g$.

Conclusion. We studied stiff directed lines (SDLs) in $1+d$ dimensions subject to quenched short-range random potential analytically and numerically. Using Florytype scaling arguments and a replica calculation we show that, in dimensions $d>2 / 3$, a localization transition ex- ists from a high temperature phase, where the system is essentially annealed, to a disorder-dominated low temperature phase. The low temperature phase is characterized by large free energy fluctuations with an exponent $\omega>0$, which cannot be calculated by scaling arguments or replica calculations. By extensive numerical transfer matrix calculations in $1+1$ dimensions we find a value $\omega \approx 0.18$, which is close to the established value $\omega \approx 0.186$ for directed lines (DLs) under tension in $1+3$ dimensions. Moreover, the rescaled free energy distributions are identical. Both points suggest that the nature of the low-temperature phase is very similar, if not identical.

This strongly supports a relation between DLs in $1+3 d$ and SDLs in $1+d$ dimensions, which is based on identical return exponents $\chi$ for two replicas to meet. The validity of a relation based on properties of a single replica pair suggests that the critical properties of DLs in a shortrange random potential are governed by replica pair interactions. The mapping can make DL transitions in high dimensions computationally accessible, which we demonstrated in showing that the two-replica overlap provides a valid order parameter across the localization transition of SDLs in 1+1 dimensions. Furthermore, the importance of pair interactions suggests that the critical temperature for DLs in random potentials is indeed identical to the temperature below which the ratio of the second moment of the partition function and the square of its first moment diverges. The binding transition of DL pairs becomes discontinuous for $d>4$ and, analogously, the binding of SDL pairs for $d>4 / 3$ [16, 34]. Because DLs in random potentials are equivalent to the KPZ equation [2, the validated relation to the SDL suggests that the roughening transition of the KPZ problem could acquire similar discontinuous features for $d>4$ dimensions. Finally, we calculated the reduction of the 
persistence length of a stiff directed by disorder.

We acknowledge financial support by the Deutsche
Forschungsgemeinschaft (KI 662/2-1).
[1] T. Halpin-Healy and Y.C. Zhang, Phys. Rep. 254, 215 (1995).

[2] M. Kardar, G. Parisi, and Y.C. Zhang, Phys. Rev. Lett. 56, 889 (1986).

[3] J. Krug, Adv. Phys. 46, 139 (1997).

[4] G. Blatter, M.V. Feigelman, V.B. Geshkenbein, A.I. Larkin, and V.M. Vinokur, Rev. Mod. Phys. 66, 1125 (1994).

[5] T. Nattermann and S. Scheidl, Adv. Phys. 49, 607 (2000).

[6] B. Derrida and O. Golinelli, Phys. Rev. A 41, 4160 (1990).

[7] J.M. Kim, A.J. Bray, and M.A. Moore, Phys. Rev. A 44, R4782 (1991).

[8] J.M. Kim, M.A. Moore, and A.J. Bray, Phys. Rev. A 44, 2345 (1991).

[9] C. Monthus and T. Garel, Eur. Phys. J. B 53, 39 (2006).

[10] E. Katzav and M. Schwartz, Physica A 309, 69 (2002).

[11] R.A. Harris and J.E. Hearst, J. Chem. Phys. 44(7), 2595 (1966).

[12] O. Kratky and G. Porod, Recl. Trav. Chim. Pays-Bas 68(12), 1106 (1949).

[13] A. Dua, T. Vilgis, J. Chem. Phys. 121, 5505 (2004).

[14] H. Kleinert, Path Integrals in Quantum Mechanics, Statistics, Polymer Physics, and Financial Markets (World Scientific, Singapur, 2006).

[15] R. Bundschuh, M. Lässig, and R. Lipowsky, Eur. Phys. J. E 3, 295 (2000).

[16] J. Kierfeld and R. Lipowsky, J. Phys. A 38, L155 (2005).

[17] M.E. Fisher, J. Stat. Phys. 34, 667 (1984).

[18] G. Gompper and T.W. Burkhardt, Phys. Rev. A 40, 6124 (1989).

[19] C. Monthus and T. Garel, Phys. Rev. E 75, 051122 (2007).
[20] R. Bundschuh and M. Lässig, Phys. Rev. E 54, 304 (1996).

[21] S. Mukherji and S.M. Bhattacharjee, Phys. Rev. B 53, R6002 (1996).

[22] S. Mukherji, Phys. Rev. E 50, R2407 (1994).

[23] M. Mezard, J. Phys. Frace 51, 1831 (1990).

[24] F. Comets, T. Shiga and N. Yoshida, Bernoulli 9(4), 705 (2003).

[25] D.S. Fisher and D.A. Huse, Phys. Rev. B 43, 10728 (1991).

[26] C. Monthus and T. Garel, Phys. Rev. E 74, 011101 (2006)

[27] P. Le Doussal and K. J. Wiese, Phys. Rev. E 72, 035101(R) (2005).

[28] V. Dotsenko, Introduction to the Replica Theory of Disordered Statistical Systems (Cambridge University Press, Cambridge, 2001).

[29] M. Mezard and G. Parisi, J. Physique I 1, 809 (1991).

[30] X. Wang, S. Havlin, and M. Schwartz, J. Phys. Chem. B 104, 3875 (2000).

[31] E. Marinari, A. Pagnani, and G. Parisi, J. Phys. A: Math. Gen. 33, 8181 (2000). The nomenclature differs: our $\omega$ is not the $\omega$ listed in this paper, but $\omega=\frac{\chi}{2-\chi}$.

[32] M. Schwartz and E. Perlsman, Phys. Rev. E 85, 050103(R) (2012).

[33] C.A. Doty and J.M. Kosterlitz, Phys. Rev. Lett. 69(13), 1979 (1992).

[34] J. Kierfeld and R. Lipowsky, Europhys. Lett. 62, 285 (2003).

[35] $\bar{X}$ denotes the quenched disorder average over realizations of $V$, whereas $\langle X\rangle$ denotes thermal averaging.

[36] We use energy units $k_{B} \equiv 1$. 